\title{
Designing for oral storytelling practices at home: a parental perspective
}

\author{
Asimina Vasalou \\ UCL Knowledge Lab \\ University College London \\ UK \\ a.vasalou@ucl.ac.uk \\ Sara Kalantari \\ UCL Knowledge Lab \\ University College London \\ UK \\ s.kalantari@ucl.ac.uk \\ Natalia Kucirkova \\ University of Stavanger \\ Norway \\ natalia.kucirkova@uis.no \\ Yvonne Vezzoli \\ UCL Knowledge Lab \\ UCL Institute of Education \\ UK \\ y.vezzoli@ucl.ac.uk
}

\begin{abstract}
Storytelling at home is typically an oral practice that supports parents and children to make sense of their family identity. Parents play a key role in crafting the story plot and facilitating the child's participation in the storytelling process. Yet in the context of digital technology, interaction design researchers have tended to focus on children and how digital storytelling tools can support them in their learning. Adopting a "family lens" the aim of this research is to understand the character of oral storytelling practice and identify opportunities for digital storytelling design. The findings outline a co-design workshop that involved a team of designers and parents, who regularly engaged in oral storytelling. Grounded in a systematic video analysis of the workshop alongside a reflection of the design decisions that unfolded, we contribute four new design opportunities centring on the themes of flexibility, shared experience, minimalism, and autobiographical memories. These opportunities can guide interaction design researchers interested in designing new digital oral storytelling tools for families.
\end{abstract}

\section{Introduction}

Storytelling is a universal human practice that expresses an understanding of our history, social relationships, and the way we see the world. Storytelling as a family practice has been the focus of several studies, with a particular emphasis on the way parents support children's understanding of the relationships and knowledge contained in stories (Doyle et al., 2006). Accordingly, digital storytelling tools have been designed to support children's social, affective, cognitive and behavioural outcomes with the adult supporting the child's learning on the side (e.g. Al-Mousawi and Alsumait, 2012; Amresh et al, 2015; Rubegni and Landoni, 2018). This past work has shown that storytelling supports children's meaning-making across different domains.

Home storytelling practices can be motivated by parents' goal to support their children's learning and print literacy (Vezzoli et al., 2020). Stories shared in the family are also verbal, or oral, whilst expressing how children and parents understand their family culture, identity and norms. Oral storytelling can be therefore understood as a social process whereby identity is negotiated and constructed among family members (Koenig Kellas, 2003). In the present work, we explore this perspective in relation to digital storytelling. We seek to 
build upon the practice of oral storytelling in the family and its socio-cultural dimensions with the aim to explore the role digital storytelling can play in the family. Our focus on family practice is in alignment with a holistic "family lens" taken in recent research within the field of child-computer interaction that highlights the importance of designing for shared family experiences (Christensen et al., 2019).

The research reported takes a design-oriented approach to prototype a new mobile technology for parent-child oral storytelling. Given the limited involvement of parents in previous design research for digital storytelling, we conducted a co-design session with parents and designers. We identify the character of oral storytelling and at the same time we engage in a reflective dialogue about the role of mobile technology to contribute four design opportunities. First, we show the importance of designing to support flexibility in relation to parents' diverse creative skills and storytelling goals. For example, while we find evidence that parents reflect their culture and norms in the stories they make and tell their children, their goal is also to support the pair's joint engagement. Second, we identify the need to design for different forms of shared experience. Not only do children occupy different roles in the storytelling process, but stories can be shared beyond the immediate family intergenerationally. Third, a tension is revealed between the time it takes to make a 'good story' and parents' time constraints suggesting the need for minimalist design that combines story making and telling in the same moment. Finally, we show the value of capturing stories and storytelling to support future autobiographical memories. These four design opportunities are informed by a reflective narrative account of our design decisions where we contrast digital storytelling for the family with digital storytelling designed for children's learning. This comparison enables us document the distinct character and concerns of oral storytelling for the home toward supporting future digital efforts to take a "family lens" on digital storytelling.

\section{Relevant work}

\section{Oral storytelling in the family}

Stories are an essentially human meaning-making tool (Bruner, 2003). Stories are embedded diachronically in family life starting from when children are very young and continuing into adulthood with books often used as prompts to facilitate storytelling (Curenton et al, 2008; Koenig Kellas, 2005). Sharing narrative stories is a key socio-cultural practice that enables children to appropriate and engage in their culture, share information, build expectations and construct memories about the past (Vygotsky, 1978). A recent study found that parent-child storytelling practices that are supported by a book are underpinned by diverse goals. These span from individual goals such as the parent's desire to support their child's language and literacy, to joint goals such as the pair's mutual need for bonding (Vezzoli et al, 2020). Therefore, unlike the role of storytelling in formal learning contexts where a learning aim directs the activity and its interactions, storytelling at home is a practice that serves multiple purposes and benefits children and parents in different ways. Most importantly, storytelling at home is multimodal with stories often shared orally as part of families' mundane everyday practices.

Oral storytelling involves stories that are orchestrated individually, or jointly, and shared between parents and children (Curenton et al, 2008). Culture plays a role in the structure and components of family stories, as illustrated by Curenton et al. (2008) through the study of oral stories shared between parents and children from different cultural groups. In contrast to the European tradition of stories that present a coherent beginning, middle and end with a main focal character, Curenton (2006) provides an example from Afro-American families who construct stories associated with multiple characters featured centrally in the stories. Furthermore, in Curenton's study, the act of composing oral stories was a symmetrical exchange by parents and children from various cultural groups. In contrast, other work shows that there is often an asymmetry between the directive nature of parents' prompts and its impact on children's narratives (e.g. Peterson and McCabe, 1994), suggesting that children's agency during storytelling is heavily mediated by the parent.

There is a tight connection between oral storytelling and family identity, as captured by Koenig Kellas (2005): "family stories affect and reflect family culture by communicating who a family is, its norms, its values, its goals, its identity. (pg. 366)" Many family stories evolve into life narratives and stories we tell about ourselves and the place we come from, and researchers have shown how parent-child reminiscing fosters children's 
development of autobiographical memory (Fivush, 2008). In addition to its role to support family identity, family storytelling can help young children to reason about their personal identities in relation to their social interactions and interpersonal conflicts (Curenton et al, 2008). Taking a learning lens, Cutspec (2006) argues that oracy not only allows the child to actively construct a more complex understanding of the world, but it also fosters children's motivation for print literacy, as such providing an additional pathway to the child's learning.

\section{Children and digital storytelling}

Despite the importance of oral storytelling at home, digital storytelling tools have predominantly been viewed as a way to facilitate children's literacy outcomes and have been often designed to be used in a formal education context aligning with existing curricula. Children's use of digital storytelling authoring has been found to improve children's learning outcomes such as communication (Al-Mousawi and Alsumait, 2012), emotion regulation and rehearsal of emotions (Wallbaum et al., 2017) as well as understanding of specific concepts, such as for example healthy eating (Amresh et al, 2015). Interaction designers have grappled with balancing children's creative open-ended story construction with a highly scaffolded story-making process designed to foster specific outcomes. Wallbaum et al (2017) identify this tension between structure and creativity asking how much agency children should have in story creation. Amresh and colleagues (2015), for example, addressed this in their digital comics by predefining cause-effect scenarios designed to facilitate discussions about food choices and their health consequences. In other work, Pittarello and Bertani (2012) created a storytelling authoring tool with different story structures and a library of prompts meant to steer children to express the individual elements of their story (e.g. weather, time, characters).

Several studies have documented how children's stories, especially when these stories are created collaboratively, can entail a creation, sharing and performance phase (Göttel et al., 2011). Most of the research to date has focused its attention on how to design technology that can scaffold the first, story creation phase. A key opportunity that mobile technology affords, and one that has been explored in previous work, is the link between children's situated experiences and story creation. Accordingly, a number of digital storytelling tools provide children with the facility to collect audio or photographs of their environments and experiences, and use these 'prompts' as inspiration for later creation of their stories (e.g. Fails et al., 2010; Halloran et al., 2006; Pittarello and Bertani, 2012). To capitalise on this affordance, it is not surprising that previous work has tended to opt for portable technology, such as mobile phones or tablets, when designing digital storytelling tools. Moreover, offering the ability to construct stories multimodally - e.g. through audio, pictures, text - has been shown to support children with different profiles to create stories (Bonsignore et al, 2013).

In summary, oral storytelling in the family is a pervasive multifaceted practice that supports learning, identity and bonding in the family. The majority of past research on digital storytelling has focused on the role of interaction design in supporting the child's learning during the story creation process, with less attention dedicated to how technology can support the whole process of storytelling including sharing and performance. Though mainly focusing on how digital tools can support story creation, one exception is Bosignore et al (2013) who co-designed the storytelling application 'StoryKit' with children and also their parents in a series of workshops. However, as the authors reflect, despite their design intensions, StoryKit mostly appealed to the needs of school teachers. Thus, there is a continued need to place the everyday oral storytelling home practice at the centre of design thinking to understand and problematise how storytelling technology can be designed for this context.

\section{Methodology}

\section{Research through design for oral storytelling at home}

Following a design thinking approach, our initial research aims centred on shared reading between parents and children. At the onset, we recognized that much of the past research in book reading has tended to take a learning focus informed by literature in early literacy and child development. Accordingly, the reading activity has been viewed as one that benefits the child with scaffolding from the adult (e.g. Doyle et al., 2006). However, we also recognised that reading is part of a family's intimate life as it brings both parent and child physically together to strengthen their affective bond. We thus took the view that reading is an intimate and 
socially constructed act. We applied an exploratory lens to understand the meaningful dimensions of this social practice, alongside any tensions parents and children experienced when reading books together, in order to identify new opportunities for digital technology design that supports shared reading (see Vezzoli et al., 2020).

In seeking to understand this perspective empirically, we used the cultural probes design method, which allowed us to gain access to families' reading practices at home. Our focus was on the child-parent pair, and we recruited six different families. With the exception of one, the families were multilingual. The cultural probe method consisted of three creative tasks asking the child and parent to express how a focal book supported their individual and shared enjoyment. Once families completed the tasks, there was a short interview. This research approach allowed us to capture the physical closeness of the parent-child pair when reading, the intimate and quiet place chosen for book reading, and the facilitative role the parent played in the child's engagement and language exposure in the book. We identified that children actively sought those moments from their parents and that parents valued them. However, it was difficult for the parents to take on the role of the storyteller and orchestrate these moments given their busy everyday lives. Our exploratory research led us to formulate the following design question: How might we support busy and tired parents to take on the role of a caring and entertaining storyteller in an intimate space?

Grounded in this design question, our five-person design team ran an ideation workshop to generate creative ideas around this theme. Amongst the different ideas that were proposed was a "template for story from the head", which emphasised how parents engaged in storytelling as a spontaneous and creative oral practice. This idea moved us away from storytelling around a book to recognize the prevalence, cultural importance and inclusivity of oral stories. We identified a gap in interaction design research in relation to the design of digital technology for oral storytelling practices in the home context. The literature in oral storytelling showed that it is a dynamic co-constructed act that strengthens family identity and also supports the child to negotiate their personal identity. As identified in the literature, parents play a central role in orchestrating, mediating, and delivering oral stories to their young children. Having identified a potentially important design idea, we planned a co-design workshop between designers and parents to create a new prototype for family oral storytelling. Whereas children have been previously involved in co-designing storytelling technology, we wanted to ground our design in the everyday home practice of oral storytelling and therefore it was important to foreground the parental perspective. As such, we involved parents in the creative process of generating solutions that were better fits to the identified design problem (Steen et al., 2011; Kristensson et al., 2002).

Design-oriented research produces its own knowledge contributions ranging from new ways of framing problems, to conceptualization of theory, critical accounts of technology and design artefacts (Gaver, 2012; Zimmerman et al, 2007). The aim of this paper was to reflect on how technology can support the socio-cultural practice of oral storytelling at home, by identifying new considerations that may guide further design in this area. In having reframed the way parent-child shared reading and storytelling has been viewed in the past within the context of digital technology, our focus was to develop a new critical understanding of this practice in order to inform design opportunities. In line with this, we report the results from our prototyping phase following Shön's (1987) reflective account of design. As Shön explains "the designer constructs the design world within which he/she sets the dimensions of his/her problem space, and invents the moves by which he/she attempts to find solutions." Building on this view, we document our unfolding design decisions, in particular, recognising moments where our decisions created conflict and uncertainty. Moreover, aligned with the reflective and iterative process of our design thinking, we critique and compare the design insights in relation to relevant literature.

Figure 1 summarises our design practice and research. First, we engaged with literature on parent-child shared reading and bonding. This perspective was enriched with empirical data from cultural probes resulting in a design question that reflected the broader parental experience of storytelling. After engaging in creative ideation to address the design question, we moved away from storytelling with physical books and identified the significance of oral storytelling for family identity. This led us to rescope our literature review away from shared reading to oral storytelling and digital storytelling tools informing the next prototyping phase. 


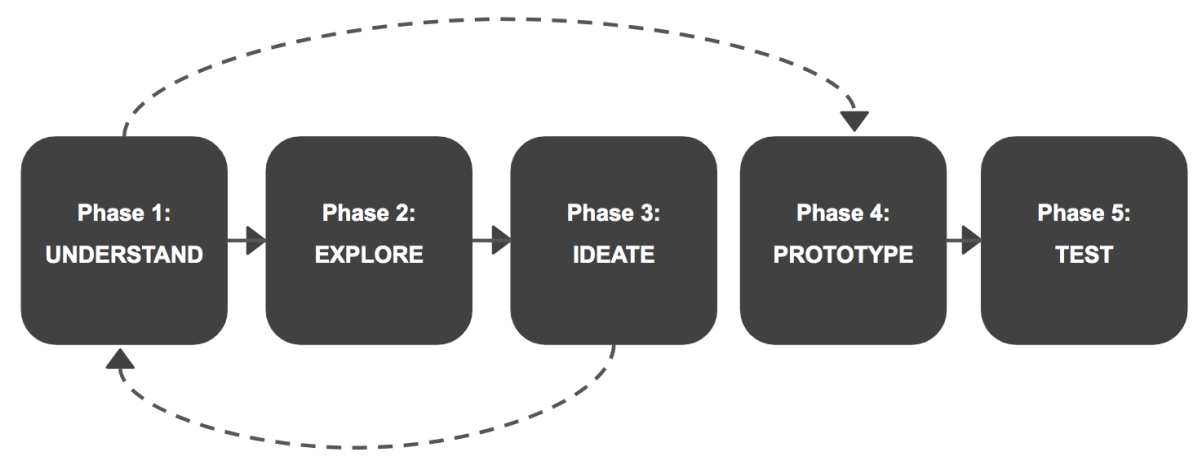

Figure 1. Our non-linear design-oriented research phases

\section{Participants}

Four parents of children aged 5-7 years old (P1-P4) and four designers from (D1-D4) participated in the prototyping workshop. All of the parents reported practicing oral storytelling at home with their children alongside other literacy and language activities and the designers had know-how on how to engage in creative design. There were also two researchers $(\mathrm{R} 1, \mathrm{R} 2)$ present at the workshop. One researcher was in charge of facilitating the workshop, while the other took observational notes. The facilitator role entailed bringing participants up to date with the state of our design research, presenting the workshop agenda, and providing step-by-step guidelines and supportive material throughout the session. By occasionally asking questions without contributing any ideas of their own, the researchers also ensured the participants understood the objective of each task and stayed on focused on the task. The workshops took place in a big seminar room with each team occupying a table.

Table 1 - Participant demographics

\begin{tabular}{|l|l|l|l|l|}
\hline Participant & Gender & Age & Occupation & $\begin{array}{l}\text { Number of } \\
\text { Children }\end{array}$ \\
\hline P1 & F & 40s & Associate Professor & 1 \\
\hline P2 & F & 40s & Psychologist & 2 \\
\hline P3 & F & 40s & Architect & 2 \\
\hline P4 & F & 40s & Associate Professor & 2 \\
\hline D1 & M & 30s & $\begin{array}{l}\text { Creative Game } \\
\text { Design Director }\end{array}$ & 1 \\
\hline D2 & F & 30s & $\begin{array}{l}\text { Postdoctoral } \\
\text { researcher }\end{array}$ & 0 \\
\hline D3 & M & 40s & Lecturer & 0 \\
\hline D4 & F & 20s & Game Designer & 0 \\
\hline
\end{tabular}

\section{Workshop Structure and Activities}

In recent decades the practice of design has moved away from focusing on "making of stuff" to a focus on "making stuff for people in the context of their lives" (Sanders, 2013; pg. 64). So that we do not only grasp a better understanding of these parent-participants' current life contexts, but also help them imagine and communicate alternative futures, it was essential to provide participants with means of conceptualisation 
beyond stuff (ibid, emphasis added). As such, the workshop activities and their supportive material were planned in such a way to allow the participants to tell their own stories around their existing practices of storytelling with the goal to visualise future enhancements of these practices through building low-fidelity prototypes.

The workshop consisted of five different activities: interviewing, storyboarding, journey mapping, prototyping and sharing (see Figure 2). For the first activity, interviewing, the participants were split into four parentdesigner pairs. The pairs later merged to form two teams of four (with an equal number of parents and designers) for the remainder of the session. Overall, the workshop lasted four hours, with intermittent breaks. We hereby provide a more detailed description of the structure of the workshop to illustrate the step-by-step process in generating a low-fidelity prototype for digital storytelling that builds on parental practice and looks at storytelling more holistically.

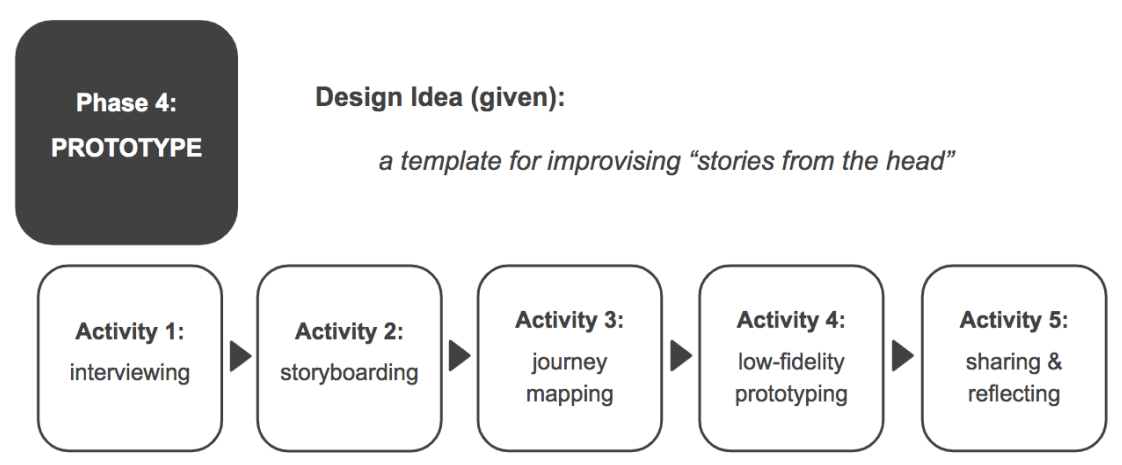

Figure 2. Prototyping phase of our research process, consisting of 5 activities

\section{Activity 1: Interviewing}

Participants were grouped into four pairs, each pair consisting of one parent and one designer. The designers were provided with a list of seven interview questions for inspiration, but were also encouraged to come up with their own questions to pose to the parents in their designated groups. The aim of the interview was to understand parents" existing experience with oral storytelling referred to as "telling stories from their heads". Some example suggested questions were:

- Does your child ever ask you to tell them oral stories ("stories from your head")?

- What type of stories do you usually create and how do you currently come up with stories?

- Does improvising come to you easily?

- Aside from fulfilling your child's desire, do you have any goals when telling "stories from your head"?

The purpose of this exercise was to introduce the conceptual focus of the workshop, but also - and perhaps more importantly - to generate some first-hand insights about the practice of oral storytelling that the participants could work with during the following activities.

Activity 2: Storyboarding

For this activity, and all remaining ones, two participant pairs were combined to form two teams of four, with half the members of each team consisting of parents and the other half designers. Each team was then given 5 minutes to come up with a "story from their heads" and another 15 minutes to "tell" the story in a visual format using a storyboard template handout provided to them. The aim of this exercise was for the participants to gain empathy with potential users of their prototype when confronted with the task of improvising a story from scratch. A secondary intention was to document the different types of stories the parents in each team might be telling their children when asked for "a story from the head", thus providing a shared understanding of storytelling practices. Drawing from P2, and her improvised storytelling, Figure 3 illustrates a story the team together crafted of a lost sheep that was adopted by a little boy as a pet. 


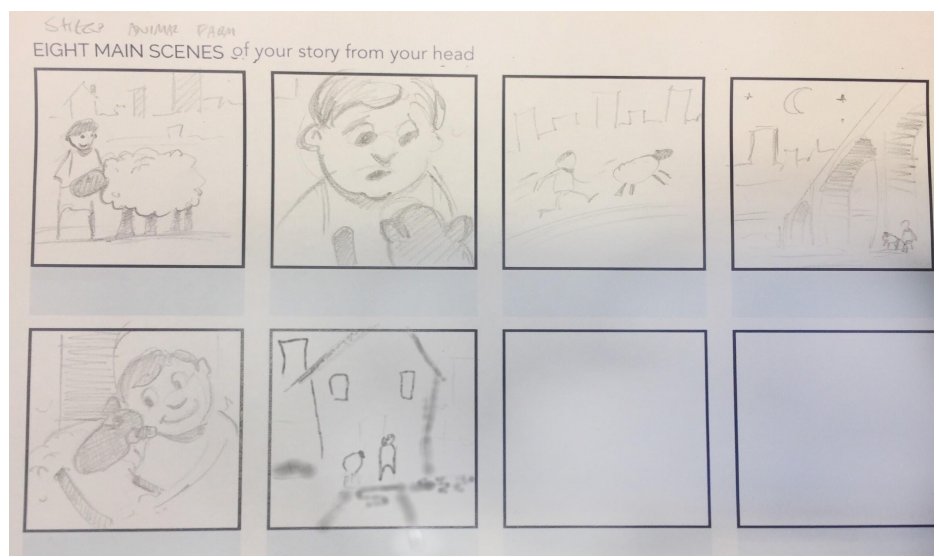

Figure 3. An example storyboard created by participants

\section{Activity 3: Journey Mapping}

After storyboarding, the teams were asked to consult their interview results and reflect on when the journey of creating a "story from the head" began for them. We challenged them to think before and beyond the creation phase of the story, from the story's moment of conception all the way to its sharing and delivery. They were encouraged to use journey map templates handed out to them in order to jot down any noteworthy, or relevant experience points, they encountered along the way in their current experience of oral storytelling (Figure 4). They were further instructed to use coloured stickers to identify rewarding (green) and challenging (red) experiences throughout their journey. The intended outcome of this activity was to help participants become aware of any existing processes that they might currently be following, whether implicitly or explicitly, in coming up with their own stories and performing them. By further marking their experience points as positive or negative, participants could later turn them into opportunity moments or points of tension to be resolved when designing their prototypes.

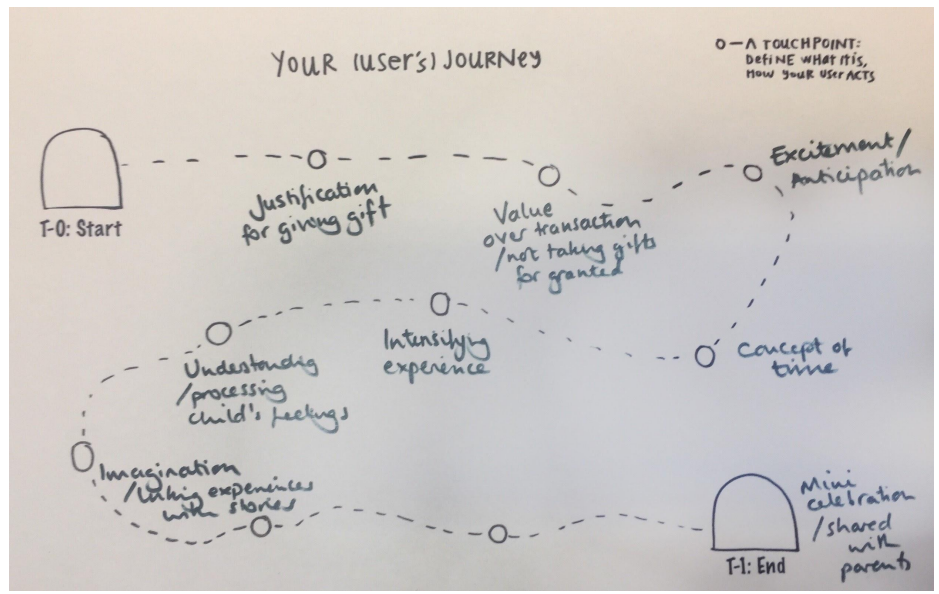

Figure 4. An example journey map created by participants

\section{Activity 4: Prototyping}

Whereas the motivation behind the previous three activities was to capture parents' current experience and practice of oral storytelling without the help of technology, the aim of the next activity was to imagine how digital technology like an application on a tablet or smartphone might help users with improvising and delivering oral stories. Following past design approaches (e.g. Bonsignore et al, 2013; Fails et al., 2010; Halloran et al., 2006; Pittarello and Bertani, 2012), and in order to cater to any "a-ha" moments encountered anywhere along the story journey, we envisioned a portable digital technology offering space to author stories on the go; hence the decision to focus on mobile screen-based technologies.

Having prepared our participants to articulate and share the practices of oral storytelling in the first workshop phases, we encouraged them to brainstorm and visualise digital solutions that could help them in creating, sharing and perhaps re-telling of oral stories in the future. Each team was provided with blank smartphone and tablet templates along with printed icons and "buttons" as optional material to sketch their imagined solutions. Using these materials, participants "built" or sketched their prototypes iteratively until finally one prototype from each team was selected for show-and-tell in the following activity. (Figure 5). 


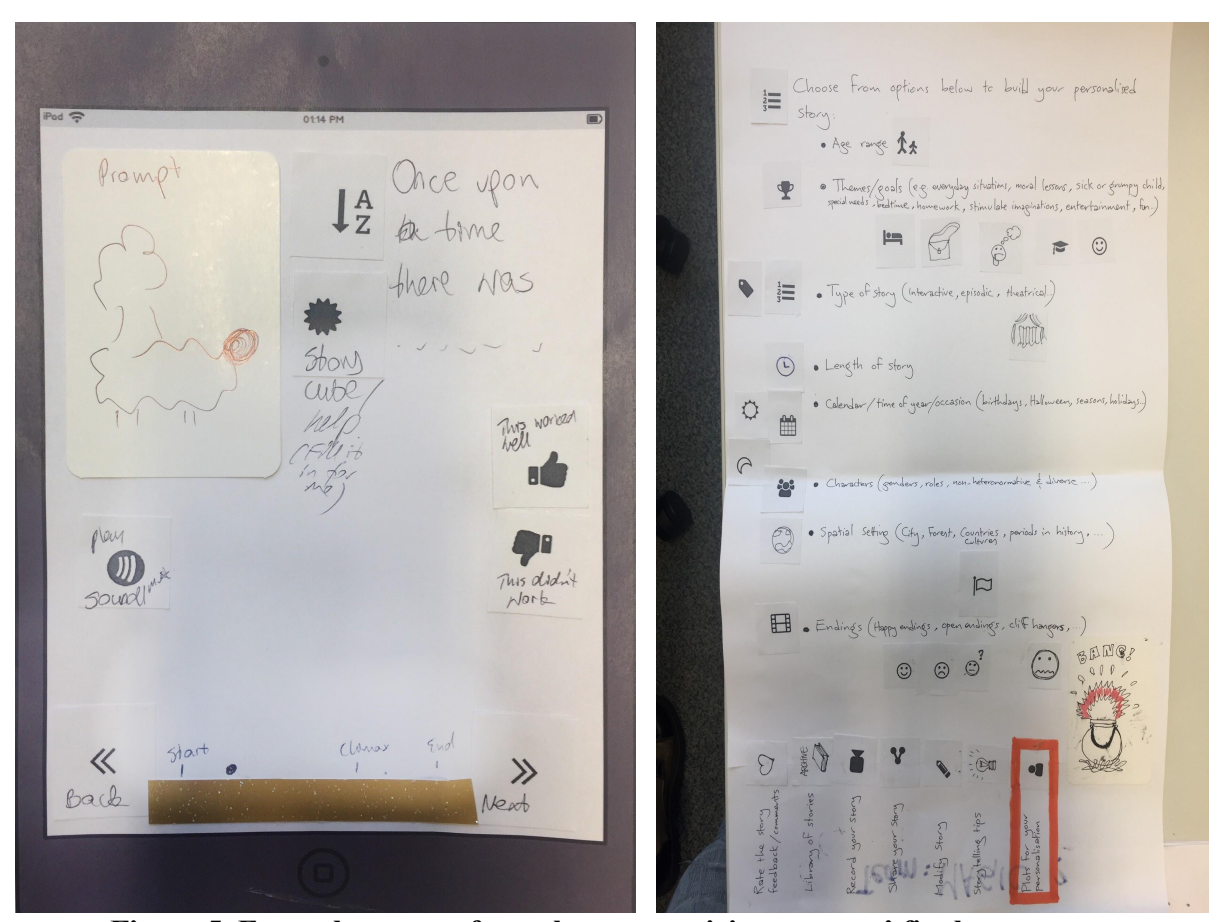

Figure 5. Example screens from the two participant teams' final prototypes

\section{Activity 5: Sharing and Reflecting}

The final stage was for each team to present their final prototype and to receive feedback from the other participants. The floor was then opened up to a general discussion, during which the teams reflected on their joint practices and design decisions made, facilitating a critical narrative about the choices made. Participants were also asked to reflect on their overall experience with the workshop and appreciate the relevance of each activity.

\section{Data Collection and Analysis}

In addition to the field notes taken by one of the researchers, with the consent of the participants, the entire workshop was audio recorded and later transcribed. With the exception of activity 1 (i.e. interviewing), all other activities were additionally video recorded at a team-level and later annotated for marking any noteworthy deviations from or augmentations to the audio transcripts. All design artefacts created by participants were also collected and scanned or photographed for archiving. By triangulating between the created artefacts, audio and video transcripts, as well as our field notes, we were able to paint a more complete picture of each team's thought processes, trials and tribulations when it came to deciding what to include or eliminate in their final prototypes, and the reasons why. Following the Collaborative Qualitative Analysis process described by Richards and Hemphill (2017), two researchers coded the transcripts inductively, using open coding to identify discrete concepts and patterns in the data. After comparing the results, a consensus was reached as to which codes and subthemes were most relevant to the overarching theme of the practice of co-constructing oral stories. A codebook was thus created from these relevant codes and applied to the rest of the data in an iterative fashion, taking note of any new codes that emerged. After coding all the data and realising that both participant teams had created similar prototypes, a decision was made to report on all interview transcripts but to focus our analysis mainly on design reflections of Team 1.

\section{Design reflections from the workshop}

\subsection{Personalized story themes and parental goals}

During the workshop interviews and the hands-on activities, parents shared a number of different approaches to their storytelling. P1 narrated stories her father had shared with her as a child. Living away from her native country, she hoped these stories would bring her son closer to the culture and history of her family and his grandparents. As she explained: “...they tend to be stories from my parents' lives. And childhood specifically, so kind of innocent stories that have some kind of underlying moral to them." Her son asked to listen to these stories once the bedtime book had been read, extending the time the pair shared together and indicating the 
child's care for his mother's heritage: "It tends to happen when my son doesn't want the bedtime story to end. And he knows that I like to share these kinds of stories with him, so he knows these are important to me. That's when he's usually prompting me to tell the story."

Taking a different approach to oral storytelling, P2 engaged in improvised storytelling anchored in her children's relationships, and the interpersonal challenges between them. Her hope was to facilitate her children's reflection on their conflicts, seeking in part to engender perspective-taking between her older and younger daughter. P2 nurtured a tight connection between the story and her child's life, and thus her children sometimes approached her with requests to tell stories about relationships they wanted to relive: "My older daughter, she was already 11, she would come to me and say, tell me a story about the (our) dog. And then I'd create the story for her."

P3 also improvised, but used children's classical stories to devise her stories. She recognized children's dual need for repetition and novelty. Therefore, she engaged in different renditions of the same story, always adapted to fit her children's age. P3 engaged in this practice to stimulate her children's imagination by drawing on stories they enjoyed. At the same time, the ability to control the timeline of a story provided her with more control over her children's bedtime, often shortening the story when she wanted her children to sleep. She explained:

"I wanted to get them to sleep quite quickly, so I would take Cinderella story and abbreviate it, and stick my own stuff in it. No, it was a story I knew, I just made it on my own... Like a summary. And it was good because I didn't have to look for books and do all the... cause when you do a kids' story, you have to repeat things. Most of kids' books have a lot of repetition for an adult is like "oh my god, do I have to say all of that again?" So if you, I found that it's less boring if you put your own kind of stuff in it."

Some of these story themes aligned with previous research. Stories were seen as a way to strengthen the family identity, particularly through sharing intergenerational values and heritage (Koenig Kellas, 2005). Stories were also crafted to support children's understanding of their relationships within their family (Curenton, 2008). Alongside these identity-oriented storytelling practices, we identified a novel practice: one in which the parents approached existing classical stories as an opportunity for sharing creative renditions intended to maintain the joint engagement of both parent and child. Reflecting on Curenton's (2006) research, in particular, who show that story structure can differ between families, the story examples shared by the three parents all followed the European tradition of story structure in that there were some living story characters, there was a main storyline and story climax. Despite the structural similarity of the stories, however, it is notable that each parent had a consistent but yet very different goal when engaging in oral storytelling with their children. Parents' desire to promote family heritage on one hand, and social skills on the other, emphasised the importance of supporting the diversity of this family practice. This created a rich conceptual space that the team returned to during the prototyping session.

\subsection{Improvisation aids for parents' creativity}

When sharing their practices, it was evident that parents acted as the main orchestrators of the storytelling process, either by creating the story or by supporting their child to co-create it. 'Prompts' have previously been designed to support children's story creation by providing scaffolds for making the story (e.g. Pittarello and Betrani, 2012) as well as enabling children to create authentic connections with their experiences (e.g. Fails et al., 2010). During the workshop, prompts were redefined as a mechanism to facilitate parental creativity in the process of story creation. We recognized that parents have different creative skills when it comes to improvising oral stories. For example, P1 shared a worry about lacking creativity in the story creation moment, and P3 described a sense of creative fatigue during storytelling. Aligned with this problem definition, we drew on a repertoire of existing interaction design techniques known to foster creativity in story creation. Seeking to support the design of a story beginning and subsequent events, we envisioned the possibility of parents to take photographs of notable moments, or objects throughout their day to be later used in the story creation process as 'personalized prompts' for improvisation. Alongside the use of these 'personalized prompts', another core idea that emerged was the value of 'pre-authored prompts' of characters and events available within the prototype to 
engender creative topic association: "so having something that just like randomly gave you prompts... you could press a button and it would just throw out a sheep, a radiator or a dragon..." (D1).

Supporting the parent to put together the main building blocks of the story through 'prompts' was seen as critical to story improvisation, as to the structure of the story. D1 explained: "So part of the reticence to improvising stories is filling the head to follow a structure. So there's lots of classical story structures, like hero journey type stories, that you could have as basic templates. So you start with this, and then, so there's a sheep and then the template would come to something to throw the sheep out of his compass and send him off in a funny direction, and so that would guide you to an ending, basically. You can have very simple versions of story structures." A number of 'story templates' were subsequently proposed based on different story genres - e.g. moral, comedy, action, tragedy, nonsensical -as a way to support parents' memory. At the same time as recognizing the value of scaffolding parents' memory, we reflected on the role these 'story templates' could play in facilitating parents' confidence in creative storytelling by 'breaking the barriers' through the availability of concrete story genre examples.

Flexibility became a guiding principle when defining how 'prompts' and 'story templates' should be designed to support parents in the act of story creation. Whereas these aids could help parents who lacked confidence or expertise in improvisation, we did not want to impose them on all parents, given that some parents, for example, P2, had developed her own creative practice. Storytelling technologies for children have been designed to always feature story making aids, such as prompts, in order to scaffold the child during the task. In contrast, storytelling technology designed for parents introduced the need to adjust these aids depending on the parents' creative skills. Moreover, we aspired for 'story templates' to support the diversity of identity and bonding practices we found parents to carry out through oral storytelling. D1 explained: "the main one for me was that people have very different goals at times when they're telling a story. And then P1 particularly, she was very interesting as she was conveying stories of her heritage, of her grandparents and ... which is not necessarily a natural thing, at least to me, to do. But when I think about it that's really a very valuable thing to be doing. And so I think any sort of template needs to be kind of flexible and to allow for the different things." Storytelling technologies for children have sometimes taken a highly structured approach that sought to balance children's creativity with the need to create stories that foster pre-defined learning outcomes (e.g. Wallbaum et al, 2017). In contrast, whilst designing technology that allows the parent to give the initial momentum of the oral story, we recognized the role of parents' agency in shaping story themes to support their children in their own ways. This could be reconciled in digital technology through the option to choose from a number of different 'story templates'.

\subsection{Participation and shared experience}

Prompted by P1's creative concern over delivering a compelling story for her child, D1 highlighted that a good story is not only tied to the content, but it is shaped by the experience shared in the moment between parent and child. Throughout the design workshop, the participation of the child in the storytelling moment was a central concern, and at times was viewed as a key ingredient to a shared parent-child experience. This aligns with a socio-cultural view on children's learning (Wegerif et al., 1999), and literature that encourages joint participation around digital media to facilitate parent-child engagement and the child's learning (Yen et al., 2018). Yet, given the different ways in which parents at the workshop described their storytelling traditions at home, the role of the child and consequently, the shared parent-child experience was very different for each dyad.

P1 and her child shared a story that foregrounded the mother's wish to connect to the past and the pair's engagement in their joint heritage. P1 was responsible for narrating the story, as the child quietly listened. Their joint experience was centered in imagining the lives and experiences of their family members. Similarly, P3 retold classic stories her children enjoyed, calibrating the story to keep them both jointly engaged. She remained the main narrator, with her children interrupting only when the story was not faithfully told. This contrasted with P2 who encouraged her children to co-create the story with her. Both parent and child took turns evolving the story with the parent taking responsibility for the facilitation of co-creation: "So whenever I would interject in the middle of the story, I would kind of give, if you want, a kind of climax, that would somehow turn, well eventually, into an ending. " Over time, P2's children adopted an agentic role during the 
story creation process: “... but they would interject as well and say (things like), 'this can happen now', or 'no, no, this is too sad'." Shared experience in this family emerged out of these interactions, and the conceptual space the family shared: "I was amazed at how they picked it up! You know, I would have something in my mind and then it comes out from my daughter's mouth." While co-creating their story, the family imbued it with shared values, resulting in a tight sense of intersubjectivity and family identity.

These diverse practices showed the situatedness of storytelling: as a malleable practice by the parent, the child's role could change depending on the parent's goal. As D3 pointed out: “...you may really be into co-creation, but another parent might want to tell a story about something that happened to the grandfather and don 't want the child to spin it into a funny direction." Therefore, the principle of flexibility also extended to the role of the child. D4 summarized the design implication: "(we want) the possibility to draw children's participation, but at the same time to be able to tell stories without this aspect". At the same time as recognising this diversity, in our own design work, we drew inspiration from P2 to conceive the inclusion of children during the collection of everyday photographs in the form of 'personalized prompts' offered alongside those generated by their parents. This was envisioned to encourage children's active participation during story creation, which as P1 argued could additionally facilitate parents' creativity. Children's participation was also promoted with the idea of 'dialogic prompts' reminding parents to invite their child's contribution at critical moments within the story e.g. 'what do you think will happen next?' Despite aligning with past work that promotes the co-creation of stories between parents and children (e.g. Troseth et al., 2019), our workshop also emphasised the importance of avoiding a normative account of child-parent roles when designing for oral storytelling practice at home (cf. Yen et al., 2018). Indeed, important values - such as maintaining the family's cultural identity, or orchestrating a quiet moment - were accomplished when the child was positioned as a passive listener.

Our team was mainly preoccupied with the shared experience between the child and the parent. However, throughout the workshop, we constantly returned to P1's storytelling practice to extend ideas originally designed for the child-parent pair to also apply to intergenerational storytelling. We recognized that families are often geographically separated. All three families represented at the workshop lived far away from their grandparents, whilst sometimes they, or their spouses, travelled for work. The idea of 'prompts' was opened up to include 'a shared family store' of contributions from family members who were far away. We envisioned children using these 'shared prompts' in their stories, provoking new questions about their family members that bring them emotionally closer together. Whereas 'shared prompts' invited the child into the lives of others, our team reflected on the value of sharing children's stories (as constructed with 'shared prompts') with the extended family, in turn inviting them into the life of the child. In her everyday practice P1 reinforced her relationship with the past through reminiscing; this was achieved by sharing with her child past events of the grandparents' lives. In contrast, our team's design thinking did not engage with the past as it focused on strengthening family relationships in the present moment by fostering shared story making experiences. Previous work in this area has focused on supporting child-grandparent dialogue around a print book as a way to develop their intimate relationship at a distance (e.g. Raffle et al., 2010). Our design workshop suggests there is also an opportunity for geographically separated children and grandparents to jointly construct stories. In alignment with Bonsignore et al (2013), who observed collocated story making by children and their grandparents, we suggest that such joint story-making can act as a way to reinforce family bonding. Moreover, given the importance our parent participants placed in promoting the culture and heritage of their children's grandparents, digital story making could also play a future role in maintaining and strengthening intergenerational family values.

\subsection{The time and place of technology}

In analysing the character of design thinking, Dorst (2006) argues that design paradoxes can emerge when the designer faces conflicting criteria that require a redefinition of the design problem and solution. His example of a design paradox is a chair that is needed to play a function at one time, but also needs to disappear at another moment as it takes up physical space. During the workshop, we faced two design paradoxes sparked by the practice of oral storytelling at home.

The first paradox reflected a tension around the time dimension of the storytelling act. All three parents explained that stories were created and shared with their children in the same moment. In the words of P2, "The 
reality of parenting is that you are so busy during the day that for me the process of making a story is what actually works there and then". The unrehearsed nature of oral storytelling practice and the lack of a linear process separated in time created a conflict with the staged model of storytelling (creation-sharingperformance) designed into past digital authoring storytelling apps (e.g. Pittarello and Bertani, 2012; Rubegni and Landoni, 2018). A tension thus emerged between the need to support parental creativity prior to story sharing, and the importance to abandon the staged approach to storytelling in order to maintain the ephemeral character of oral storytelling. Instead of reconceptualising the design of our story creation mode, this challenged us to redefine the notion of story representation, i.e. the fidelity and completeness of creating stories with digital technology. We envisioned parents using the improvisation aids to design story outlines, as opposed to polished stories, which would provide an anchor for parents to later share richer verbal story renditions of the story.

Though oral storytelling practice led us to acknowledge the importance of unifying the three story phases, during the workshop we also identified the opportunities technology could offer to the oral storytelling practice by separating the three phases in time. Created stories could be saved within the app and later accessed by the parent and child to support new renditions of story performance, reflecting the practice taken by P3. As D1 pointed out: "I guess there's the story as it was told, and then there's the template of that story that you might wanna tell the same plot. Cause you know there's a separation between the plot and the actual telling of the story. I might wanna tell you the story of Little Red Riding Hood afresh saying in a different way but it follows the same structure." In summary, our workshop revealed a misalignment between the time potentially required by a digital tool to create stories featuring a high level of representation and the ephemeral storytelling practice at home. We also identified the importance of creating stories that leave space for parents and children to engage in different story renditions in the future. Unpacking the dimensions of this socially-constructed practice highlighted the importance of minimalist design for story making that on the one hand, supports parents to maintain short-lived engagements in story creation, whilst the same minimalism also allows children and parents to bring their own meaning when sharing and performing the story (Kaye et al., 2005).

Our team discussions centred on how technology can support the practice of oral storytelling in the present moment for diverse families but we also considered the opportunity for technology to facilitate the creation and retrieval of autobiographical memories for children and their parents. We envisioned the capability of recording aspects of the story creation and sharing process in order to later replay them. Thus, whilst digital stories offer a context of constructing a shared experience in the present, stories can be archivable objects of remembrance to offer precious mementos of the family's past intimate moments. This aspect of story-sharing in families plays an important role in fostering family (collective) as well as individual (the child's personal) identity, and the voice-recording in multimedia apps can facilitate this process, as recognized by our participants:

\section{D2: And we talked about these voice recorders to capture... because it was a co-created story, so to capture} different voices and be able to play them back to children later, a few years later even, so that they can....

P2: I love it!

D1: Yeah, we said the same thing...I think for parents as well, when parents listen to their kid's voice and say "oh my god, I remember when you would say ..."

The second design paradox involved a tension between time/place of storytelling and the presence of technology. Past research has shown that storytelling with books can often take place at bedtime (Vezzoli et al., 2020; Chang and Breazeal, 2011), a tendency we noticed during our conversations with the workshop participants. The time and place of storytelling conceptualized by the participants defined the activity as an intimate moment whose intimate character needed to be preserved. It fostered children's connection with their family culture, served as a calming gesture of care, and involved a dialogic exchange between parents and children. Since both story creation and sharing phases were characteristic of oral storytelling, the presence of technology during the act of sharing was seen as both valuable and disruptive. The facility to record and reminisce on oral story sharing was unanimously embraced by all three parents. However, there was a concern that technology would take attention away from the story sharing, and overstimulate the child leading to the requirement to hide away the technology. This tension remained unresolved during the workshop. 
P2: Yeah, so when we're recording, I think that the recording thing... of that...I'd say act as a front thing...I would use it discreetly and not maybe pass it on to my daughter and say 'OK, now add something', cause then she would want to play a game and she would wake up... and I think my recording would be made discreetly, discreetly recording their voice and maybe sometimes very discreetly taking a photo. But that's it. I wouldn't really involve them with the digital...

P1: So I wonder actually, whether this is, there's this opportunity moment because effectively what you were saying is that in the moment of sharing the stories with the child, the screen might have a lesser role to play, so...

P2: Yes, but before the night you have the story probably, or you have all the information you want. You as a parent look at it, you have it there and then you will retract the digital

P3: You can still take the device with you in the room but just hide it, put it in an out of reach and out of sight mode. Make the recording happen in as passive a way as possible.

\subsection{Design Opportunities for Digital Storytelling in the Home Context}

So far we described the practice and character of oral storytelling in the family, alongside the emergent design considerations and tensions we encountered when designing a prototype to support this practice. We now draw on the reflective analysis of our design process to summarise four design opportunities for digital storytelling tools designed to support oral storytelling in the family. Each opportunity is grounded in findings from the workshop, whilst Table 2 captures illustrative ideas that emerged in the co-design workshop to exemplify possible design solutions for each design opportunity.

Flexibility in supporting parents' diverse practices

Our findings showed that oral stories are orchestrated by parents. Some parent-participants did not have the confidence, or the creative skills, to craft original oral stories. In contrast, others were natural storytellers and were able to improvise stories in the moment. In recognising the parent's role in the story creation process, digital storytelling could offer 'on demand' improvisation aids to parents who need the support. Further to the need to flexibly support parents with different creative profiles, we identified the importance of supporting parents to enact their different storytelling goals, which were shaped based on different criteria e.g. the child's needs, the family culture as well as the parent's values. Digital storytelling could be thus designed to afford parents agency for the type of story they want to develop without constraining them to follow a pre-given story genre, or story goal.

Promoting different forms of shared experience

Our findings showed that each parent's distinctive approach to oral storytelling shaped how their child participated. Whereas two of the parent-participants controlled the delivery of the story creation and telling, a third parent co-created the story with their children. Our workshop highlighted that the level of interaction embedded in different child roles can be valuable in different ways. Child roles with less interaction can foster children's imagination, engagement with cultural values or knowledge, and promote quiet time between parent and child. In contrast, roles that promote more interaction can develop children's creativity and narrative skills. Digital storytelling for families could be designed to avoid prescriptive role assumptions, for example by requiring children's participation. In addition to recognising the diverse practices and child outcomes of this shared experience, we also found that shared experience can extend beyond the parent-child pair to include other family members. In particular, the parent-participants involved in the workshop identified the importance to preserve their culture and intergenerational values through involving grandparents. Digital storytelling could, therefore, allow for families to configure the members involved in story creation and sharing.

\section{Minimalism for situated practice}

Our workshop revealed that oral storytelling is a spontaneous and situated human practice in which the parentparticipants reported to create, share and perform stories for their children all at the same moment. This was because parents lacked the time to prepare pre-authored stories. This introduces a design challenge for digital storytelling, which has tended to take a staged model to storytelling. Digital storytelling could be designed to support parents and children to develop minimal stories 'in the moment' that avoid introducing new workload 
to this currently ephemeral practice. Minimal stories could open up opportunities for richer and diverse renditions of the same story during story sharing and performance, thus recognising the vital role of the storyteller in enriching what is not captured in the digital story representation.

\section{Stories fostering autobiographical memories}

Our findings identified the need to nurture reminiscence between the parent-child pair. Digital storytelling could, therefore, support autobiographical memories for the parent-child, for example by offering them the option to archive their stories and record the process of making them. Archiving the content of a story can foster reminiscence of the child's life including their learning, relationships and heritage. Moreover, recordings of parents and children's story co-creation process can later convey the dynamic and negotiated identity process that makes up a family.

\begin{tabular}{|c|c|c|}
\hline Design opportunity & Example ideas from the workshop & $\begin{array}{l}\text { Mapping } \\
\text { to sections }\end{array}$ \\
\hline $\begin{array}{l}\text { Flexibility in supporting } \\
\text { parents' diverse practices }\end{array}$ & $\begin{array}{l}\text { Provide pictures, i.e., 'pre-authored prompts', to support parents } \\
\text { in creative associations } \\
\text { - Enable parents to collect pictures during their day, which can } \\
\text { later serve as 'personalised prompts' used to spark their creativity in } \\
\text { story creation } \\
\text { - Offer spines of different story genres that parents can select } \\
\text { before engaging in story creation }\end{array}$ & $4.1,4.2$ \\
\hline $\begin{array}{l}\text { Promoting different forms of } \\
\text { shared experience }\end{array}$ & $\begin{array}{l}\text { - Give children the option to collect pictures during their day, } \\
\text { which can later serve as 'personalised prompts' used by children and } \\
\text { their parents in story creation process } \\
\text { - Offer 'on demand' dialogic scaffolds parents can use to actively } \\
\text { involve their child in the story creation process } \\
\text { - Extend the idea of 'personalised prompts' to include extended } \\
\text { family } \\
\text { - Allow for stories to be shared with the extended family }\end{array}$ & 4.2 \\
\hline $\begin{array}{l}\text { Minimalism for situated } \\
\text { practice }\end{array}$ & $\begin{array}{l}\text { Provide a low entry point to story creation that promotes the } \\
\text { creation of story drafts }\end{array}$ & 4.3 \\
\hline $\begin{array}{l}\text { Stories fostering } \\
\text { autobiographical memories }\end{array}$ & $\begin{array}{l}\text { - Recording the stories created, shared and performed by parents } \\
\text { and children }\end{array}$ & 4.3 \\
\hline
\end{tabular}

Table 2. Design implications and example ideas from the co-design workshop

\section{Conclusion}

Oral storytelling is a frequent practice in the family. It fosters the family's shared understanding of their values and norms, provides a context for children to reflect on their personal identities and relationships, as well as creates a context for bonding. While past work in the field of interaction design has treated storytelling as an opportunity to scaffold children's learning typically through the application of evidenced-based principles from education, in this work we have taken a different perspective. We argued that technology designed to facilitate oral storytelling in the family should also recognise the broader and more diversified role that storytelling plays in family life, as well as build upon the characteristics of oral storytelling practices at home. We wanted to foreground the parental perspective in oral storytelling practices, which has been so far unexplored. Whilst applying this lens, we took a design-oriented research approach and carried out a co-design workshop during which parents and designers prototyped new technology for oral storytelling in the home.

Our research has revealed the multi-faceted role that oral stories can play in family life and has raised four new considerations for designing digital storytelling: flexibility in relation to supporting parents' diverse creative profiles and storytelling goals; shared experience by recognising the value of children contributing to stories as well as listening, and extending storytelling to foster intergenerational shared experiences; designing minimalist technology that concurrently supports story creation and telling; and archiving stories and recording story creation to later support autobiographical memories. In taking a "family lens" that recognises oral storytelling practice, the new design opportunities are markedly different to those previously identified when designing digital storytelling tools for children's learning. Our study therefore offers a new set of concerns to guide future 
digital storytelling tools designed for the context of the home. The co-design workshop findings we reported captured important dimensions of oral storytelling practices in families that were in turn generative. However, alongside our small scale design research, we hope future research in this area can contribute to a more expansive understanding of parent-child oral storytelling practices in the home context. Moreover, though we have shed some light on the valuable role digital tools could play to support oral storytelling we must recognise the unresolved complexities. In particular, oral stories were often told during bedtime and ensured the family had quiet time together (Vezzoli et al., 2020; Chang and Breazeal, 2011). The need to maintain the thoughtful space and time of oral stories stands in contrast to the introduction of mobile technology, and the child's immersion or distraction away from the shared experience with the parent, and thus remains an unresolved challenge.

\section{ACKNOWLEDGEMENTS}

This research was funded by the EPSRC project 'Dynamic, Real time, On-demand Personalisation for Scaling (DROPS)' under EP/R033838/1. We thank the designers and parents for their participation, and our DROPS project partners for their continuing feedback on our research. We are grateful to Elisa Rubegni and the reviewers of this paper whose comments have improved our work.

\section{REFERENCES}

[1] Ashish Amresh, Rahul Salla, Madhumita Sinha and Rebecca A. Birr. 2015. Interactive Cause and Effect Comicbook Storytelling for Improving Nutrition Outcomes in Children. In Proceedings of the 5th International Conference on Digital Health 2015, 9-14. https://doi.org/10.1145/2750511.2750533

[2] Bruner, J. S. (2003). Making stories: Law, literature, life. Harvard University Press.

[3] Angela Chang and Cynthia Breazeal. 2011. TinkRBook: Shared Reading Interfaces for Storytelling. In Proceedings of the 2011 Conference on Interaction Design and Children, 145-148.

https://doi.org/10.1145/1999030.1999047

[4] Peter Knøsgaard Christensen, Christoffer Øland Skovgaard, and Marianne Graves Petersen. 2019. Together Together: Combining Shared and Separate Activities in Designing Technology for Family Life. In Proceedings of the 18th ACM International Conference on Interaction Design and Children (IDC '19). Association for Computing Machinery, New York, NY, USA, 374-385. DOI:https://doi.org/10.1145/3311927.3323141

[5] Stephanie M. Curenton, Michelle Jones Craig and Nadia Flanigan. 2008. Use of Decontextualized Talk Across Story Contexts: How Oral Storytelling and Emergent Reading Can Scaffold Children's Development. Early Education \& Development, 19, 1, 161-187. http://dx.doi.org/10.1080/10409280701839296

[6] Stephanie M. Curenton. 2006. A cultural art that promotes school readiness. Young Children, 78-89.

[7] Patricia A. Cutspec. 2006. Oral storytelling within the context of parent-child relationship. Talaris Research Institute, $1,2,1-8$.

[8] Doyle, B. G., \& Bramwell, W. (2006). Promoting emergent literacy and social-emotional learning through dialogic reading. The Reading Teacher, 59(6), 554-564.

[9] Jerry Alan Fails, Allison Druin and Mona Leigh Guha. 2010. Mobile collaboration: collaboratively reading and creating children's stories on mobile devices. In Proceedings of the 2010 Interaction Design and Children Conference, 2029.

[10] Robyn Fivush. 2008. Remembering and reminiscing: How individual lives are constructed in family narratives. Memory studies, 1, 1, 49-58.

[11] Timo Göttel. Reviewing Children's Collaboration Practices in Storytelling Environments. In Proceedings of the 2011 Interaction Design and Children Conference, 97-100.

[12] John Halloran, Eva Hornecker, Geraldine Fitzpatrick, Mark Weal, David Millard, Danius Michaelides, Don Cruickshank, David De Roure. The Literacy Fieldtrip: Using UbiComp to Support Children's Creative Writing. In Proceedings of the 2006 Interaction Design and Children Conference, 17-24.

[13] Jody Koenig Kellas. 2005. Family Ties: Communicating Identity Through Jointly Told Family Stories. Communication Monographs, 72, 4, 365-389. https://doi.org/10.1080/03637750500322453

[14] Carl Peterson and Allyssa McCabe. 1994. A social interactionist account of developing decontextualized narrative skill. Developmental Psychology, 30, 6, 937. https://doi.org/10.1016/S0959-4752(99)00013-4

[15] Fabio Pittarello and Luca Bertani. CASTOR: Learning to create Context-Sensitive and emotionally engaging narrations in situ. In Proceedings of the 2012 Interaction Design and Children Conference, 1-10.

https://doi.org/10.1145/2307096.2307098 
[16] Elisa Rubegni and Monica Landoni. 2018. Fiabot! Design and Evaluation of a Mobile Storytelling_Application for Schools. In Proceedings of the 2014 Conference on Interaction design and children.

https://doi.org/10.1145/2593968.2593979

[17] Georgene L. Troseth, Gabrielle A. Strouse, Israel Flores, Zachary D Stuckelman, Colleen Russo Johnson. 2019. An enhanced eBook facilitates parent-child talk during shared reading by families of low socioeconomic status. Early Childhood Research Quarterly. https://doi.org/10.1016/j.ecresq.2019.02.009

[18] Yvonne Vezzoli, Sara Kalantari, Natalia Kucirkova and Asimina Vasalou. 2020. Exploring the design space for parent-child reading. In Proceedings of the 2020 Conference of Human Factors in Computing Systems (In Press).

[19] Torben Wallbaum, Swamy Ananthanarayan, Shadan Sadeghian Borojeni, Wilko Heuten and Susanne Boll. 2017. Thematic Workshops. https://doi.org/10.1145/3126686.3126702

[20] Kate Yen, Yeqi Chen, Yi Cheng, Sijin Chen, Ying-Yu Chen, Yiran Ni, and Alexis Hiniker. 2018. Joint Media Engagement between Parents and Preschoolers in the U.S., China, and Taiwan. In Proceedings of the ACM in HumanComputer Interaction, 2., Article 192 (November 2018), 19 pages. https://doi.org/10.1145/3274461

[21] Al-Mousawi, and Alsumait. 2012. A digital storytelling tool for Arab children. In Proceedings of the 14th International Conference on Information Integration and Web-based Applications \& Services, 26-35. https://doi.org/10.1145/2428736.2428746

[22] Donald Schön. 1987. Educating the Reflective Practitioner. Jossey- Bass, San Francisco CA.

[23] John Zimmerman Jodie Forlizzi, and Shellyy Evenson. 2007. Research through design as a method for interaction design research in HCI. In Proceedings of the SIGCHI conference on human factors in computing systems , 493-502.

[24] Steen, M., Manschot, M., \& De Koning, N. (2011). Benefits of co-design in service design projects. International Journal of Design, 5(2), 53-60.

[25] Kristensson, P., Magnusson, P., \& Matthing, J. (2002). Users as a hidden resource for creativity: Findings from an experimental study on user involvement. Creativity and Innovation Management, 11(1), 55Sanders, E. B. N. 2013. "Prototyping for the Design Spaces of the Future." In Prototype: Design and Craft in the 21 st Century, edited by Louise Valentine. London: Bloomsbury.

[26] Elizabeth Sanders 2013. "Prototyping for the Design Spaces of the Future." In Prototype: Design and Craft in the 21 st Century, edited by Louise Valentine. London: Bloomsbury.

[27] Elizabeth Bonsignore, Alexander J. Quinn, Allison Druin, and Benjamin B. Bederson. 2013. Sharing Stories "in the Wild": A Mobile Storytelling Case Study Using StoryKit. ACM Trans. Comput.-Hum. Interact. 20, 3, Article 18 (July 2013), 38 pages. DOI: https://doi.org/10.1145/2491500.2491506

[28] Hayes Raffle, Rafael Ballagas, Glenda Revelle, Hiroshi Horii, Sean Follmer, Janet G, Emily Reardon, Koichi Mori, Joseph 'Jofish' Kaye, Mirjana Spasojevic. 2010. Family Story Play: Reading with Young Children (and Elmo) Over a Distance. In Proceedings of the SIGCHI Conference on Human Factors in Computing Systems, 1583-1592. https://doi.org/10.1145/1753326.1753563

[29] Joseph "Jofish” Kaye, Mariah K. Levitt, Jeffrey Nevins, Jessica Golden, and Vanessa Schmidt. 2005. Communicating intimacy one bit at a time. In CHI '05 Extended Abstracts on Human Factors in Computing Systems (CHI EA '05). Association for Computing Machinery, New York, NY, USA, 1529-1532. DOI:https://doi.org/10.1145/1056808.1056958

[30] Kevin Andrew Richard and Michael A. Hemphill. A practical guide to collaborative data analysis. Journal of Teaching in Physical Education 37, 2, 225-231. https://doi.org/10.1123/jtpe.2017-0084

[31] Vygotsky, L. (1978). Interaction between learning and development. Readings on the development of children, 23(3), 34-41.

[32] William Gaver. 2012. What should we expect from research through design?. In Proceedings of the SIGCHI conference on human factors in computing systems, 937-946. 